\title{
Non-inferiority evaluation of preservative-free latanoprost/timolol eye drops solution versus preserved latanoprost/timolol eye drops in patients with high intraocular pressure and open-angle glaucoma
} \author{
Lida Kalantzi 10 (iD \\ ${ }^{1}$ Attikon University Hospital, Athens, Greece \\ ${ }^{2}$ General University Hospital of Thessaloniki AHEPA, Thessaloniki, Greece \\ ${ }^{3}$ Ophthalmiatreio Athens, Athens, Greece \\ ${ }^{4}$ General Hospital of Larissa, Larissa, Thessaly, Greece \\ ${ }^{5}$ IASO Thessalias, Larissa, Greece \\ ${ }^{6}$ General University Hospital of Patra, Patra, Greece \\ ${ }^{7}$ General Hospital of Thessaloniki Ippokrateio, Thessaloniki, Greece \\ ${ }^{8}$ BECRO, Athens, Greece \\ ${ }_{9}^{9}$ NIMTS 417 Hospital, Athens, Greece \\ ${ }^{10}$ Pharmathen S.A., Attica, Greece
}

Panos Theodosiadis ${ }^{1}$, Anastasios Konstas ${ }^{2}$, Ioannis Halkiadakis ${ }^{3}$, Vasiliki Dimera ${ }^{4}$, Dimitrios Koufakis ${ }^{5}$, Constantinos D Georgakopoulos ${ }^{6}$, Evgenia Kanonidou ${ }^{7}$, Elias Zintzaras ${ }^{8}$, Michael Lygeros ${ }^{9}$, Konstantina Soulele ${ }^{10}$, Antonios Margaritis ${ }^{10}$,

\begin{abstract}
Background: This study aimed to evaluate the non-inferiority and safety of a newly developed preservative-free (PF) multi-dose latanoprost/timolol ophthalmic solution, compared with the benzalkonium chloride (BAK)-preserved fixed combination, in patients with openangle glaucoma and ocular hypertension.

Methods: A Phase III randomized multi-center observer-blind parallel-group clinical trial was conducted. A total of 210 adult patients (aged over 18 years) were randomly treated with the PF- or the BAK-preserved latanoprost/timolol solution once daily in the affected eye(s) for 12 weeks. Follow-up visits were scheduled at weeks 2, 6, and 12; intraocular pressure (IOP) was recorded at 8:00 AM, 12:00 PM, and 4:00 PM. The primary efficacy endpoint to prove non-inferiority was the IOP change at 8:00 AM ( \pm 1 hour) from the baseline to the end of treatment (week 12) in the studied eye. Safety parameters were also assessed.

Results: In total, 196 patients completed the study. The pressure-lowering effect of the PF eye drops was comparable to that of the preserved formulation at all-time points. Latanoprost/timolol PF formulation was non-inferior to the BAK-preserved solution as shown by the change in IOP from day 0 to week 12 . The point estimate of the inter-treatment difference was $0.624 \mathrm{mmHg}(95 \% \mathrm{Cl}:-0.094,1.341)$. Both treatments were well-tolerated during the study, and they had similar adverse event profiles.

Conclusions: PF-latanoprost/timolol combination was found to be non-inferior to the BAK-preserved formulation based on the efficacy at all times, with similar local tolerance.
\end{abstract}

\section{KEY WORDS}

glaucoma, intraocular pressure, benzalkonium chloride, benzalkonium, latanoprost, timolol, preservative-free, xalacom, eye drop

Copyright (C) 2020, Author(s). This is an open-access article distributed under the terms of the Creative Commons Attribution-Non Commercial 4.0 International License (http://creativecommons.org/licenses/by-nc/4.0/) which permits copy and redistribute the material just in noncommercial usages, provided the original work is properly cited.

Correspondence to: Dr. Lida Kalantzi PhD, Head of Scientific Affairs, Attica, Greece, Tel.: +30 $21066 \quad 04300$ (ext.1541). E-mail: lkalantzi@pharmathen.com

How to cite this article: Theodosiadis P, Konstas AG, Halkiadakis I, Dimera V, Koufakis D, Georgakopoulos CD, Kanonidou E, Zintzaras E, Lygeros M, Soulele K, Margaritis A, Kalantzi L. Non-inferiority evaluation of preservative-free latanoprost/timolol eye drops solution versus preserved latanoprost/timolol eye drops in patients with high intraocular pressure and open-angle glaucoma. Med Hypothesis Discov Innov Ophthalmol. 2020 Winter; 9(4): 255-263, DOI: https://doi.org/10.51329/mehdiophthal1411 


\section{INTRODUCTION}

Open-angle glaucoma is a progressive optic disc neuropathy that has become one of the most prominent causes of irreversible vision loss globally [1]. Glaucoma affects more than 70 million people globally, with a prevalence of $3.5 \%$ in patients aged 40-80 years [2, 3]. Clinical studies have confirmed the significance of high intraocular pressure (IOP) in the development and progression of this disease [4]. The decrease in this modifiable ocular parameter continues to be the cornerstone of glaucoma treatment, with topical medications still being the first-line option for the disease [1, 5].

Prostaglandin analogs and $\beta$-adrenergic receptor blocking agents have become the mainstay of glaucoma treatment due to their increased potency and safety [6]. In this respect, the fixed combination of latanoprost, the prostaglandin analog, and timolol, a $\beta$-adrenergic blocker, is approved for the treatment of open-angle glaucoma and ocular hypertension in patients with glaucoma that is not sufficiently controlled with monotherapy [7].

Latanoprost, an IOP-lowering medication, is a widely used prostaglandin analog that acts primarily by increasing the outflow of aqueous humor, whereas timolol decreases aqueous humor formation in the ciliary epithelium [7]. The two drugs have shown additive IOP-lowering effects when administered together, with several short- and long-term clinical trials validating the favorable efficacy and safety profile of their topical combination [8,9].

Xalacom $^{\text {TM }}$ (Pfizer, Kent, UK), a fixed latanoprost/timolol combination, was first approved in the EU in 2000 and is now available in several countries [1]. The marketed multi-dose formulation contains benzalkonium chloride (BAK), a commonly used and highly effective antimicrobial agent with a broad range of activities, as a preservative [5, 6]. Studies have shown that the non-specificity of the antimicrobial activity of BAK may result in tolerability issues in some cases [6]. BAK, a quaternary ammonium, may be responsible for cases of corneal epithelial cellular dysfunction, conjunctival inflammation and fibrosis, and disorders of deeper ocular tissues associated with long-term treatment $[4,10]$. Results from clinical studies have demonstrated a higher incidence of such effects in the presence of BAK, with remission of symptoms following the withdrawal of the preservative [11, 12].

In light of these effects, several topical antiglaucoma medications have been developed as preservative-free formulations over the last few years. Different beta-blockers, prostaglandin analogs, and fixed combination products have been commercialized as preservative-free formulations and marketed mainly as single-dose units [13]. A preservativefree formulation of a fixed latanoprost/timolol combination has also been marketed recently and is available in sterile unit-dose vials [14]. However, the relatively higher cost and the increased potential of mishandling these formulations, leading to an increased risk of contamination, have been reported [13].

In this respect, novel multi-dose preservative-free formulations of high quality that are easy to use have been developed [15]. One such product is the newly developed preservative-free latanoprost $50 \mu \mathrm{g} / \mathrm{mL}+$ timolol $5 \mathrm{mg} / \mathrm{mL}$ eye drop solution (Pharmathen S.A., Athens, Greece), which is packaged in a multi-dosage container with the Aero Pump $3 \mathrm{~K}$ technology to provide adequate contamination protection over repeated use through its specialized filtration and valve systems. To compare the efficacy and safety profiles of this novel preservative-free latanoprost/timolol fixed-combination and the BAK-preserved formulation $\left(\right.$ Xalacom $\left.{ }^{\mathrm{TM}}\right)$, a non-inferiority phase III study involving patients with open-angle glaucoma and ocular hypertension was conducted.

\section{METHODS}

\section{Study design and participants}

This was a national multicenter randomized referencecontrolled observer-blind non-inferiority parallel-group phase III clinical trial comparing the preservative-free ophthalmic product of latanoprost $50 \mu \mathrm{g} / \mathrm{mL}+$ timolol 5 $\mathrm{mg} / \mathrm{mL}$ eye drops solution (Pharmathen S.A., Athens, Greece) with the marketed BAK-preserved Xalacom ${ }^{\mathrm{TM}}$ (Latanoprost $50 \mu \mathrm{g} / \mathrm{ml}+$ timolol $5 \mathrm{mg} / \mathrm{mL}$, Pfizer, Kent, UK) eye drop solution in patients with open-angle glaucoma and ocular hypertension over 3 months. The study was conducted at eight clinical sites in Greece (Ophthalmiatreio Athens, General Hospital of Larissa, General University Hospital of Athens Attikon, General University Hospital of Thessaloniki AHEPA, IASO Thessalias, General University Hospital of Patra, General Hospital of Thessaloniki Ippokrateio, and NIMITS 417) between June 2018 and December 2018. The study protocol was prospectively approved by the National (Hellenic) Ethics Committee (NEC) (October 31, 2018) and the National (Hellenic) Organization of Medicines (EOF) (October 31,2018 ) and performed in accordance with the Declaration of Helsinki (2004) and Good Clinical Practice (GCP) guidelines. Before the initiation of the study, written informed consent was obtained from all participants. The study was registered in the EU Clinical Trials Register database with trial identification number 2017-004524-29.

Male or female patients aged $\geq 18$ years who (a) were diagnosed with unilateral or bilateral open-angle glaucoma or ocular hypertension, (b) had non-efficiently controlled IOP, and (c) had not used anti-glaucoma treatment for at least 4 weeks before the study initiation were included based on eligibility criteria per trial. IOP had to be between 22 and 35 $\mathrm{mmHg}$ when measured at 8:00 AM, 12:00 PM, and 4:00 PM before the treatment in at least one eye at day 0 , and the best-corrected visual acuity had to be $\geq 20$ of 100 (Snellen), which is equal to the logarithm of the minimum angle of 
resolution (LogMAR) value of 0.7 . The main exclusion criteria were as follows: history of chronic or recurrent inflammatory eye disease, ocular trauma, or infections; history of anterior chamber lens, torn posterior lens capsule, aphakia, or any known risk factor for cystoid macular edema, narrow-angle glaucoma and angle-closure glaucoma, compromised cornea or corneal abnormalities, retinal disease with clinical significance or progressive entity, intraocular surgery within the past 3 months or ocular laser surgery within the past 1 month; cup/disk ratio of $>0.8$; current use of topical, ocular, nonsteroidal anti-inflammatory drugs; history of airway or cardiovascular disease and pregnancy, breast-feeding, or childbearing potential.

The sample size of the current study was calculated to obtain the required power for the demonstration of non-inferiority of the IOP change from the baseline of the test compared to that of the control formulation. For this sample size calculation, the one-sided significance level was set at $2.5 \%$, and the power was $80 \%$. A non-inferiority margin of 1.5 $\mathrm{mmHg}$, used as the tolerance criterion, has been conventionally used and accepted in non-inferiority glaucoma studies $[16,17]$, based on the assumption of a value of 2.8 for the common standard deviation (SD) of the inter-group difference and a value of 0.3 for the true mean difference between the treatment groups. Considering these assumptions, a total sample size of 174 was used for this study, and the enrolled patients were equally and randomly allocated to the treatment groups. In addition, as the primary analysis was based on the per-protocol (PP) population, a dropout rate of approximately $20 \%$ was further assumed, resulting in a total sample size of 210 as a prerequisite for an adequately powered study (i.e., 105 patients per treatment group).

During the baseline visit (day 0), eligible patients were randomly assigned to receive the preservative-free multidose latanoprost $50 \mu \mathrm{g} / \mathrm{mL}$ + timolol $5 \mathrm{mg} / \mathrm{mL}$ fixed combination product (Pharmathen S.A.; test product group) or the BAK-preserved control formulation (Xalacom ${ }^{\mathrm{TM}}$, Pfizer; control product group) for 12 weeks. Because of the differences between the packaging of both drugs, this trial was conducted as observer-blind. Hence, the treatment allocation was conducted by personnel other than the investigator to guarantee investigator masking.

The patients were instructed to instill one drop in the affected eye(s) once daily in the evening (at approximately 8:00 PM) and scheduled for follow-up visits at weeks 2 and 6 ( \pm 2 days) and 12 ( \pm 4 days). Since the treatments were administered by the patients at home, compliance control was also performed at each visit and at the end of the treatment by visual inspection of the amount of solution in the bottle. In this respect, the patients were asked to return full and empty bottles of eye drops at the end of the clinical trial. Compliance was documented in the patient's diary. The information recorded included the date and time of each instillation, and the patient was obliged to bring the diary during every scheduled visit (weeks 2, 6, and 12).

Using a calibrated Goldmann applanation tonometer at each study visit, the IOP levels were measured and recorded by blinded evaluators twice for each eye at four-hour intervals: 8:00 AM, 12:00 PM, and 4:00 PM ( \pm 1 h). If the two measurements of the same eye differed by more than 4 $\mathrm{mmHg}$, a third measurement was performed, and the average value was used in the analysis. In patients with bilateral disease, if only one eye met the eligibility criteria (clinical trial eye), the contralateral eye was treated if it did not meet the exclusion criteria. If both eyes were eligible, the eye with a higher IOP was included. If both eyes had the same IOP, the investigator decided on the study eye.

\section{Patient Involvement}

Patients were not directly involved in the design of this study or the writing or editing of this document.

\section{Efficacy Assessments}

The primary efficacy endpoint for confirming non-inferiority was the change in IOP from day 0 to the end of week $12( \pm 4$ days) in the study eye. The mean IOP (average of two or three consecutive IOP measurements) was measured at the same time $(8: 00 \mathrm{AM} \pm 1 \mathrm{~h})$ for each visit.

The secondary efficacy variables included (i) the change in IOP at 12:00 PM and 4:00 PM in the study eye from day 0 to week 12 ( \pm 4 days), (ii) the change in IOP at 8:00 AM, 12:00 $\mathrm{PM}$, and 4:00 PM from day 0 to week 2 ( \pm 2 days), and (iii) the change in IOP at 8:00 AM, 12:00 PM, and 4:00 PM in the study eye from day 0 to week 6 .

In addition, the investigator re-evaluated the following parameters on weeks 2, 6, and 12: blood pressure, heart rate, changes in concomitant medication, visual acuity, ophthalmoscopy, intraocular pressure, ocular discomfort, adverse events, and evaluation of treatment compliance (collection of used and unused clinical trial drugs).

\section{Safety Assessments}

The evaluation of the safety parameters was based on the recordings of the ocular and systemic adverse events (AEs) along with their severity (mild, moderate, or severe) and their potential relationship with the study treatment, any clinically significant safety findings during the ocular examination, and any decrease in visual acuity from the baseline (day 0). The safety population (SP) comprised all patients who had received at least one eye drop of the preservative-free or BAK-preserved preparation.

\section{Statistical Analyses}

The principal statistical hypothesis of the study was that the new preservative-free formulation of latanoprost $50 \mu \mathrm{g} / \mathrm{mL}+$ timolol $5 \mathrm{mg} / \mathrm{mL}$ eye drops solution is non-inferior, in terms of efficacy, to Xalacom ${ }^{\text {TM }}$ (Latanoprost $50 \mu \mathrm{g} / \mathrm{ml}+$ timolol 5 $\mathrm{mg} / \mathrm{mL}$ ). In this respect, an analysis of covariance (ANCOVA) model was used to analyze the IOP change, with the 
treatment and baseline IOP as the main effect and covariate, respectively. The treatment difference and two-sided $95 \%$ confidence intervals ( $\mathrm{Cls}$ ) for the difference were calculated. The preservative-free latanoprost/timolol solution was considered to be non-inferior to the marketed BAKpreserved Xalacom ${ }^{\mathrm{TM}}$, if the upper limit for the $95 \% \mathrm{Cl}$ of the difference was $<1.5 \mathrm{mmHg}[16,17]$.

Continuous secondary efficacy variables, to be used as additional supportive evidence, were also analyzed using the ANCOVA model with the respective baseline IOP as the covariate and treatment as a factor. The treatment difference and two-sided $95 \% \mathrm{Cl}$ for the difference were also obtained for the secondary endpoints.

The demographic and baseline characteristics for the treatment groups were compared using the parametric independent t-test or the non-parametric Mann-Whitney Utest for continuous variables and the chi-squared test or Fisher's exact test for categorical variables. The safety data were compared, when appropriate, using the chi-squared test or Fisher's exact test, depending on the nature of the data. Statistical significance was set at $P<0.05$. IBM SPSS Statistics for Windows, version 24 (IBM Corp., Armonk, NY, USA), was used to conduct all analyses.

The subjects were categorized into two groups. The intentto-treat (ITT) population included all randomized patients with one post-baseline IOP measurement, whereas the perprotocol (PP) population included all subjects of the ITT population with no substantial protocol violations who completed all IOP measurements, completed at least 12 weeks of treatment, and did not take prohibited concurrent medications. Statistical analysis of the primary efficacy variable was performed for the PP population.

\section{RESULTS}

Patient baseline characteristics and disposition

A total of 210 patients were randomized into two groups and administered treatment; 106 patients received the test formulation, and 104 patients received the control formulation. Fourteen (14) patients were excluded from the PP population (test, $n=10$; control, $n=4$ ) due to consent withdrawal, adverse events, or protocol violations. Thus, 196 patients (test, $n=96$; control, $n=100$ ) were included in the PP population (Figure 1). The ITT population included 209 randomized patients, as one patient had no post-dose IOP measurements. The safety population (SP) included all randomized patients who were instilled with one dose of the study medication $(\mathrm{n}=210)$.

Table 1 shows the patient characteristics at baseline. The demographic and clinical characteristics were similar in the two treatment groups at baseline $(P>$ $0.05)$. All the recruited subjects had well-controlled IOP before inclusion in the study. The difference between the IOPs of the test and control groups during all three baseline measurements at 8:00 AM, 12:00 PM, and 4:00 PM was not significant ( $P>0.05$ for all measurements). Treatment compliance was fairly good, as the patients missed less than $20 \%$ of the planned administered doses at weeks 2, 6, and 12 (final visit) according to the patient's recordings.

Table 1. Patient characteristics and baseline data for the preservative-free (PF) test formulation (Latanoprost $50 \mu \mathrm{g} / \mathrm{ml}+\mathrm{Timolol} 5 \mathrm{mg} / \mathrm{ml}$ eye drops solution) and the BAK-preserved formulation $\left(\right.$ Xalacom $\left.^{\mathrm{TM}}\right)$, which was the control formulation for the PP population.

\begin{tabular}{|c|c|c|c|}
\hline & $\begin{array}{l}\text { Preservative-free } \\
\text { test product } \\
(\mathrm{N}=96)\end{array}$ & $\begin{array}{l}\text { BAK-preserved } \\
\text { control product } \\
(\mathrm{N}=100)\end{array}$ & $P$-value \\
\hline Age, years Mean (SD) & $69.48(11.73)$ & $70.92(10.97)$ & 0.378 \\
\hline Sex, Females, n(\%) & $52(54.2)$ & $49(49.0)$ & 0.469 \\
\hline Caucasians, $n(\%)$ & $96(100.0)$ & $100(100.0)$ & 0.999 \\
\hline Open-angle glaucoma, n(\%) & $66(68.8)$ & $71(71.0)$ & 0.731 \\
\hline Bilateral disease, $n(\%)$ & $77(80.2)$ & $82(82.0)$ & 0.749 \\
\hline Comorbidities, n(\%) & $77(80.2)$ & $78(78.0)$ & 0.704 \\
\hline History of allergies, $n(\%)$ & $3(3.1)$ & $3(3.0)$ & 0.959 \\
\hline Concomitant medications, n(\%) & $76(79.2)$ & $75(75.0)$ & 0.488 \\
\hline History of ocular surgery, $n(\%)$ & $41(42.7)$ & $43(43.0)$ & 0.967 \\
\hline History of ocular laser surgery, $n(\%)$ & $8(8.3)$ & $4(4.0)$ & 0.206 \\
\hline IOP mmHg at 8:00 AM [mean (SD)] & $24.60(2.07)$ & $25.03(2.39)$ & 0.184 \\
\hline IOP mmHg at 12:00 PM [mean (SD)] & $24.36(1.71)$ & $24.79(2.19)$ & 0.133 \\
\hline IOP mmHg at 4:00 PM [mean (SD)] & $24.37(1.85)$ & $24.44(2.00)$ & 0.813 \\
\hline
\end{tabular}

Abbreviations: BAK, Benzalkonium chloride; IOP, intraocular pressure; n, number; \%, percentage; SD, Standard deviation; PP population, per-protoco population; AM, means before midday; PM, means after midday. 


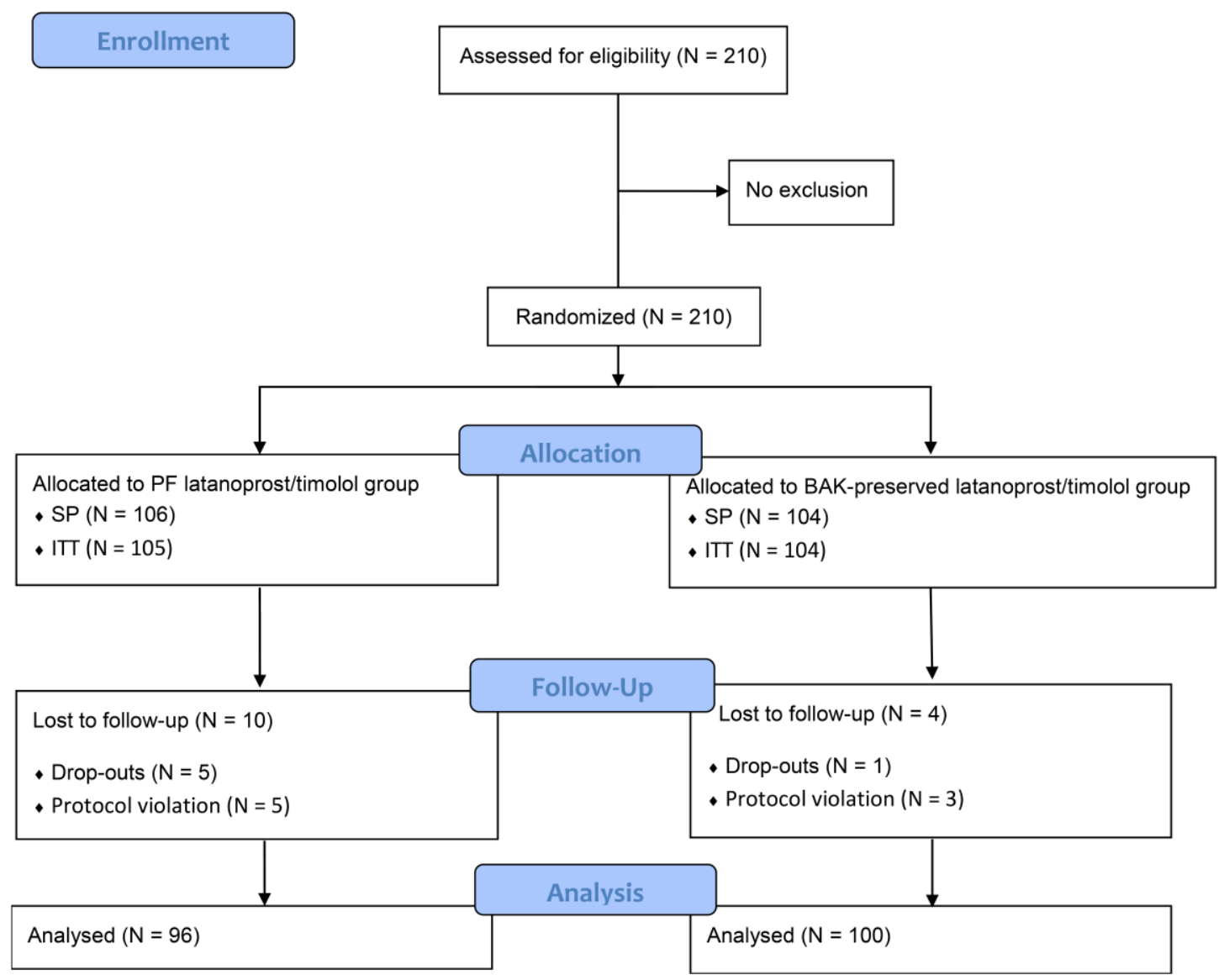

Figure 1. Disposition of patients treated with the preservative-free latanoprost/timolol formulation (Pharmathen S.A.) and the BAK-preserved control formulation Xalacom ${ }^{\mathrm{Tm}}$ (Latanoprost $50 \mu \mathrm{g} / \mathrm{ml}+$ timolol $5 \mathrm{mg} / \mathrm{mL}$, Pfizer) . Abbreviations: BAK, benzalkonium chloride; PF, preservative-free; ITT, Intent To Treat population; N, number; SP, Safety Population.

\section{Efficacy}

The non-inferiority of the test was based on the perprotocol dataset. In the PP population, the primary efficacy endpoint, which is the mean IOP change at 8:00 AM from the baseline (day 0 ) to the end of treatment (week 12), demonstrated that the preservative-free formulation was non-inferior to the existing BAKpreserved product. In particular, the mean \pm SD of the IOP decreased from $24.60 \pm 2.07 \mathrm{mmHg}$ at day 0 to $16.72 \pm 2.89 \mathrm{mmHg}$ at week 12 (final visit) in the preservative-free group and from $25.03 \pm 2.39 \mathrm{mmHg}$ to $16.37 \pm 2.90 \mathrm{mmHg}$ at the same time points for the BAK-preserved product (Table 2). The point estimate of the difference between the test and control products was $0.624 \mathrm{mmHg}(95 \% \mathrm{Cl}:-0.094,1.341)$ after adjusting for the respective baseline IOP measurements. Noninferiority was demonstrated at week 12 , as the upper limit of the $95 \% \mathrm{Cl}$ for the difference between the two treatments was below $1.5 \mathrm{mmHg}$. The statistically nonsignificant differences between the changes in IOP in the two treatment groups from baseline at all the time points for each visit were further obtained during the evaluation of the secondary efficacy variables supporting the primary efficacy analysis (Table 2). Figure 2 shows the change in mean IOP for the test (PF) and control (BAK) treatment groups, as measured at 8:00 AM during the four study visits (day 0-baseline and weeks $2,6,12$ ). These results were further confirmed by the evaluations of the ITT population. For the primary endpoint, the point estimate of the difference between the IOP changes caused by the test and control formulations at 8:00 AM in week 12 was T-C = 0.442 and the corresponding $95 \% \mathrm{Cl}$ was $(-0.271$, 1.156), after adjusting for the respective baseline IOP measurements. The difference was not statistically significant $(P=0.223)$. Thus, the upper limit $(U L)$ of the $95 \% \mathrm{Cl}$ of the difference between the two treatments ( $U L=1.156$ ) was below $1.5 \mathrm{mmHg}$, confirming the noninferiority of the PF-formulation to the BAK-preserved product. 


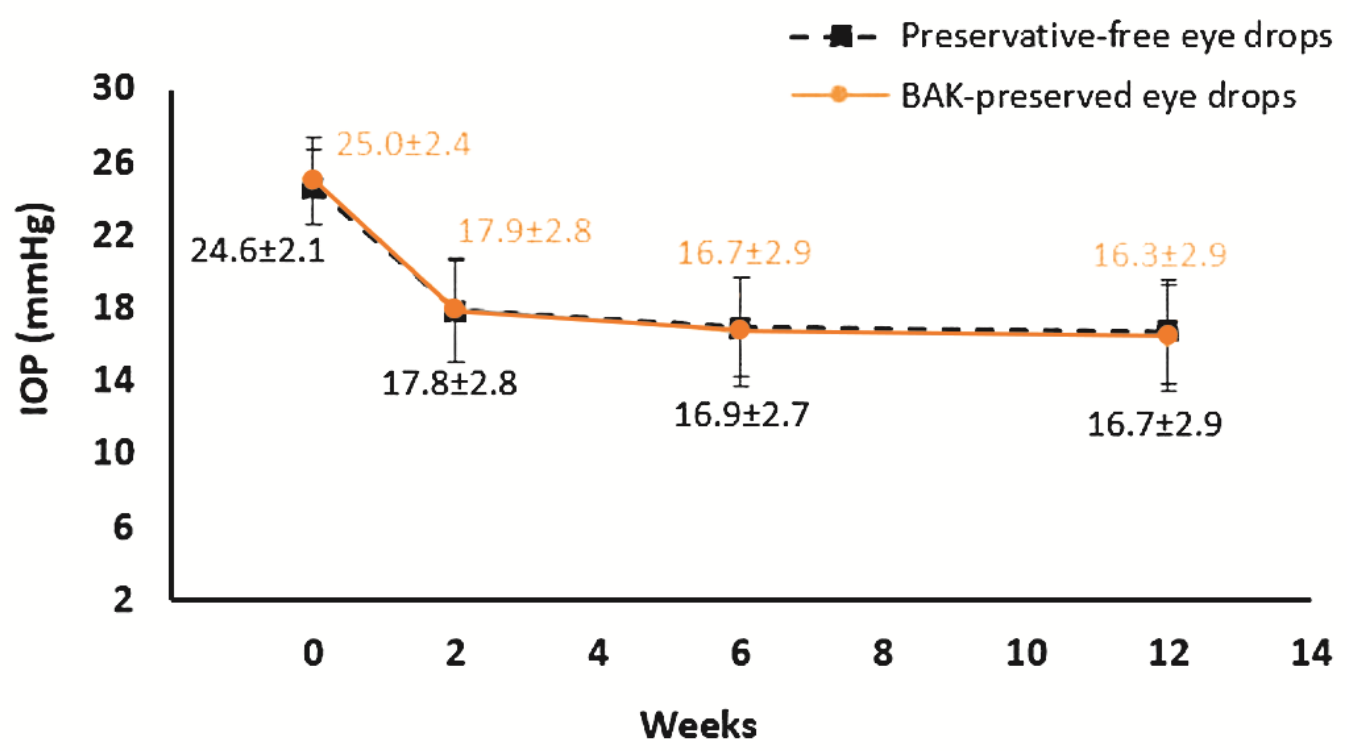

Figure 2. Change in mean IOP for the preservative-free and BAK-preserved treatment groups, as measured at 08:00 AM during the four study visits (day 0 and weeks 2, 6, 12). Error bars represent the \pm SD of the mean. Abbreviations: IOP, intraocular pressure; $\mathrm{mmHg}$, millimeters of mercury; SD, standard deviation; preservative-free eye drops, preservative-free test formulation (Latanoprost $50 \mu \mathrm{g} / \mathrm{ml}+$ Timolol $5 \mathrm{mg} / \mathrm{ml}$ eye drops solution); BAK-preserved eye drops, BAK-preserved formulation $\left(\right.$ Xalacom $\left.^{\mathrm{TM}}\right)$.

Table 2. Mean \pm SD and $95 \%$ Cls of the IOP change from baseline to the pre-specified time points for the preservative-free (PF) and BAK-preserved (BAK) formulations for the per-protocol population.

\begin{tabular}{|c|c|c|c|c|c|c|c|}
\hline \multicolumn{3}{|l|}{ Time (h) } & \multicolumn{3}{|c|}{ Mean \pm SD IOP (mmHg) } & & \\
\hline \multicolumn{8}{|c|}{ Primary efficacy endpoint } \\
\hline & \multicolumn{2}{|c|}{ Baseline } & \multicolumn{5}{|c|}{ Week 12} \\
\hline & PF & BAK & $\mathrm{PF}$ & BAK & Difference $^{a}$ & $95 \% \mathrm{Cl}^{\mathrm{b}}$ & $P$-value \\
\hline 8:00 AM & $24.60 \pm 2.07$ & $25.03 \pm 2.39$ & $16.72 \pm 2.89$ & $16.37 \pm 2.90$ & 0.624 & $-0.094,1.341$ & 0.088 \\
\hline \multicolumn{8}{|c|}{ Secondary efficacy endpoints } \\
\hline & \multicolumn{2}{|c|}{ Baseline } & \multicolumn{5}{|c|}{ Week 2} \\
\hline & $\mathrm{PF}$ & BAK & $\mathrm{PF}$ & BAK & Difference & $95 \% \mathrm{Cl}$ & $P$-value \\
\hline 8:00 AM & $24.60 \pm 2.07$ & $25.03 \pm 2.39$ & $17.83 \pm 2.79$ & $17.92 \pm 2.85$ & 0.121 & $-0.617,0.858$ & 0.747 \\
\hline 12:00 PM & $24.36 \pm 1.71$ & $24.79 \pm 2.19$ & $17.43 \pm 2.74$ & $17.71 \pm 2.83$ & 0.049 & $-0.783,0.684$ & 0.894 \\
\hline \multirow[t]{3}{*}{ 4:00 PM } & $24.37 \pm 1.85$ & $24.44 \pm 2.00$ & $17.51 \pm 2.90$ & $17.50 \pm 2.80$ & 0.044 & $-0.710,0.798$ & 0.908 \\
\hline & \multicolumn{2}{|c|}{ Baseline } & \multicolumn{5}{|c|}{ Week 6} \\
\hline & $\mathrm{PF}$ & BAK & $\mathrm{PF}$ & BAK & Difference & $95 \% \mathrm{Cl}$ & $P$-value \\
\hline 8:00 AM & $24.60 \pm 2.07$ & $25.03 \pm 2.39$ & $16.95 \pm 2.69$ & $16.71 \pm 2.97$ & 0.511 & $-0.188,1.210$ & 0.151 \\
\hline 12:00 PM & $24.36 \pm 1.71$ & $24.79 \pm 2.19$ & $16.57 \pm 2.47$ & $16.35 \pm 2.77$ & 0.471 & $-0.197,1.139$ & 0.166 \\
\hline \multirow[t]{3}{*}{ 4:00 PM } & $24.37 \pm 1.85$ & $24.44 \pm 2.00$ & $16.46 \pm 2.61$ & $16.35 \pm 2.86$ & 0.152 & $-0.572,0.875$ & 0.680 \\
\hline & \multicolumn{2}{|c|}{ Baseline } & \multicolumn{5}{|c|}{ Week 12} \\
\hline & $\mathrm{PF}$ & BAK & $\mathrm{PF}$ & BAK & Difference & $95 \% \mathrm{Cl}$ & $P$-value \\
\hline 12:00 PM & $24.36 \pm 1.71$ & $24.79 \pm 2.19$ & $16.31 \pm 2.64$ & $16.01 \pm 2.57$ & 0.569 & $-0.086,1.225$ & 0.088 \\
\hline 4:00 PM & $24.37 \pm 1.85$ & $24.44 \pm 2.00$ & $16.22 \pm 2.65$ & $15.94 \pm 2.52$ & 0.306 & $-0.392,1.003$ & 0.388 \\
\hline
\end{tabular}

${ }^{\text {aPF minus BAK; }}{ }^{\mathrm{b}}$ For all primary and secondary endpoints, the results were not significant $(P>0.05)$. Abbreviations: Preservative-free formulation, Latanoprost $50 \mu \mathrm{g} / \mathrm{ml}+$ Timolol $5 \mathrm{mg} / \mathrm{ml}$ eye drops solution; BAK-preserved formulation, Benzalkonium chloride-preserved formulation (Xalacom ${ }^{\mathrm{TM}}$ ); SD, Standard deviation; IOP, intraocular pressure; millimeter of mercury, $\mathrm{mmHg}$; $\mathrm{Cl}$, Confidence interval; \%, percentage; $\mathrm{h}$, hour; $\mathrm{AM}$, means before midday; PM, means after midday. 


\section{Safety and Tolerability}

Overall, the incidence of adverse events was low in both treatment groups, and no serious adverse events were reported for the two formulations. In total, 78 patients in the clinical trial experienced AEs. Seventy-four of those patients ( $35.3 \%$ of total patients) had ocular AEs: 34 patients were in the preservative-free group, and 40 were in the BAK-preserved group. Four patients in the PF group and one in the BAK group had systemic effects, whereas one subject presented skin and subcutaneous tissue AEs in the BAK group. The total number of reported AEs was 130: 123 were ocular, with $53(43.1 \%)$ in the PF group and 70 (56.9\%) in the BAK group (Table 3). Regarding the severity of the ocular AEs, 47 cases $(88.7 \%)$ in the PF group and $57(81.4 \%)$ in the BAK-group were considered mild, whereas $6(11.3 \%)$ in the PF group and $13(18.6 \%)$ in the BAK group were considered moderate. No clinically significant difference between the visual acuity changes caused by the treatments from baseline was observed.

\section{DISCUSSION}

In this randomized non-inferiority study, the developed preservative-free, multi-dose latanoprost $50 \mu \mathrm{g} / \mathrm{mL}+$ timolol 5 $\mathrm{mg} / \mathrm{mL}$ eye drops solution (Pharmathen S.A.) demonstrated non-inferior efficacy, compared with the marketed BAK- preserved formulation over the 12-week treatment period. A similar reduction in IOP was recorded during the first assessment (i.e., week 2) for both products, which was maintained to the end of the study (week 12). The percentage reduction in IOP from baseline to the end of week 12 was $-32 \%$ for the preservative-free solution group and $-34 \%$ for the BAKpreserved solution, corresponding to the upper limit for the $95 \% \mathrm{Cl}$ for the difference between the two treatments, which was below the non-inferiority margin of $1.5 \mathrm{mmHg}[16,17]$.

The need for the sterility of multi-dose eye drops has led to the inclusion of potent antimicrobial agents in these solutions. BAK is one of the most widely used preservatives for currently available ophthalmic solutions. It is a highly effective preservative agent with broad activity against gram-positive and gram-negative bacteria and fungi [6]. BAK is a quaternary ammonium that acts as a cationic detergent, exerting its antimicrobial activity by dissolving bacterial walls and membranes and damaging cytoplasmic contents. However, its ability to damage unicellular microbes does not seem to be confined to microbial cells; its effects may be exerted on human ocular cells as well, especially during long-term exposure [5, 18]. These effects are largely relevant for glaucoma patients who use several ophthalmic solutions multiple times per day for their lifetime [6].

Table 3. Summary of AEs for the Safety population associated with the preservative-free (PF) and BAK-preserved (BAK) formulations.

\begin{tabular}{|c|c|c|c|c|c|c|}
\hline & \multicolumn{3}{|c|}{ PF (Test, N = 96) } & \multicolumn{3}{|c|}{ BAK (Control, N = 100) } \\
\hline Deaths or Serious AEs & \multicolumn{3}{|c|}{-} & \multicolumn{3}{|c|}{-} \\
\hline Discontinuation due to $\mathrm{AE}(\mathrm{s})$ & \multicolumn{3}{|l|}{1} & \multicolumn{3}{|l|}{-} \\
\hline Total number of AEs & \multicolumn{3}{|l|}{57} & \multicolumn{3}{|l|}{73} \\
\hline \multirow[t]{2}{*}{ OCULAR AEs } & \multicolumn{3}{|c|}{53} & \multicolumn{3}{|c|}{70} \\
\hline & \multicolumn{2}{|l|}{ Mild } & Moderate & Mild & \multicolumn{2}{|l|}{ Moderate } \\
\hline Diplopia & \multicolumn{2}{|l|}{1} & - & - & \multicolumn{2}{|l|}{-} \\
\hline Eye/eyelid pruritus & \multicolumn{2}{|l|}{6} & - & 2 & \multicolumn{2}{|l|}{-} \\
\hline Eye/eyelid pain & \multicolumn{2}{|l|}{4} & - & 9 & \multicolumn{2}{|l|}{1} \\
\hline Eye dryness & \multicolumn{2}{|l|}{2} & 1 & 2 & \multicolumn{2}{|l|}{-} \\
\hline Lacrimation increased & \multicolumn{2}{|l|}{1} & - & - & \multicolumn{2}{|l|}{-} \\
\hline Burning sensation & \multicolumn{2}{|l|}{1} & - & 3 & \multicolumn{2}{|l|}{2} \\
\hline Blurred vision & \multicolumn{2}{|l|}{4} & - & 3 & \multicolumn{2}{|l|}{1} \\
\hline Application site irritation & \multicolumn{2}{|l|}{2} & - & 1 & \multicolumn{2}{|l|}{-} \\
\hline Foreign body sensation & \multicolumn{2}{|l|}{5} & 2 & 11 & 1 & \\
\hline Eye irritation & 2 & & - & - & - & \\
\hline Visual impairment & 1 & & - & 1 & - & \\
\hline IOP increased & 13 & & - & 15 & - & \\
\hline Vitreous floaters & 1 & & - & - & - & \\
\hline Conjuctival hyperaemia & 4 & & 3 & 6 & 7 & \\
\hline Abnormal sensation in the eye & - & & - & 2 & - & \\
\hline Instillation site burning & - & & - & 1 & 1 & \\
\hline Ocular hyperemia (upon waking up) & - & & - & 1 & - & \\
\hline SYSTEMIC AEs & & & & & 3 & \\
\hline & Mild & Moderate & Severe & Mild & Moderate & Severe \\
\hline Dizziness & 1 & - & - & - & - & - \\
\hline Headache & 1 & 1 & - & - & - & - \\
\hline Oedema peripheral & - & - & 1 & - & - & - \\
\hline Dermatitis & - & - & - & 1 & - & - \\
\hline Heart rate decreased & - & - & - & 1 & - & - \\
\hline Erythema of eyelid & - & - & - & 1 & - & - \\
\hline
\end{tabular}

Abbreviations: AE, Adverse events; PF, preservative-free test formulation (Latanoprost $50 \mu \mathrm{g} / \mathrm{ml}+$ Timolol $5 \mathrm{mg} / \mathrm{ml}$ eye drops solution); BAK, BAKpreserved formulation (Xalacom ${ }^{\mathrm{TM}}$ ); BAK, Benzalkonium chloride; N, number; IOP, intraocular pressure. 
In this context, several ophthalmic formulations have been developed as BAK-free or preservative-free recently [6]. Ophthalmic solutions using non-BAK preservatives, such as polyquad, sodium benzoate, and SofZia, have shown improved tolerance; however, some effects have also been observed with these antimicrobial agents. Therefore, efforts have been made to minimize the overall preservative exposure of patients, either by decreasing the number of administered drops or by decreasing or even eliminating the preservatives from these formulations [6]. In this regard, numerous preservativefree formulations are currently available, including timolol, latanoprost, the fixed combination of timolol and latanoprost, betaxolol, dorzolamide, carteolol, and tafluprost [13]. These preservative-free ophthalmic solutions have demonstrated effectiveness in controlling IOP comparable to that of BAK-preserved formulations [6], suggesting that BAK, apart from its preservative activity, does not affect the ability of the drug to lower IOP [19]. To date, clinical studies that have compared the efficacy and tolerability of BAK-preserved and nonpreserved preparations of timolol $[6,10]$, latanoprost [20, 21], carteolol [22], and tafluprost [23, 24] have demonstrated similar efficacy profiles.

Preservative-free ophthalmic solutions, however, also have disadvantages. These formulations are marketed as sterile single-dose units, and the higher cost of these dosage forms and the handling limitations leading to their contamination have been recorded [6]. To address these issues, preservative-free multi-dose formulations, which are capable of preventing contamination while maintaining the high cost-effectiveness of the product, have also been developed $[13,15]$.

The currently evaluated latanoprost $50 \mu \mathrm{g} / \mathrm{mL}+$ timolol 5 $\mathrm{mg} / \mathrm{mL}$ eye drop by Pharmathen S.A. is a multi-dose preservative-free formulation with a three-stage contamination protection system; the system includes a specially designed adsorption filter, a silver spiral at the top of the tip used to prevent bacterial growth, and a special valve system (Aero Pump 3K technology) that blocks the solution backflow hindering bacterial penetration. The current phase III study demonstrated the non-inferiority of this ophthalmic solution product to the original BAK-preserved formulation (Xalacom ${ }^{\mathrm{TM}}$, Pfizer Ltd.) in reducing IOP over 12 weeks. The current study has several limitations. Initially, this was a single-blinded study, since the differences between the packaging systems of the two products made the double-masking unfeasible. This may have affected the perception of the received treatment by the patients. Nevertheless, the masking of the principal investigator was ensured through the study procedures and appropriate training of the rest of the study staff and participants. Other potential limitations include the designation of a single-morning IOP assessment as the primary non-inferiority endpoint and the relatively short study duration, which may have prevented the coverage of the potential long-term effects of the administered treatments. However, this endpoint and study design have been well-established for the demonstration of IOP-lowering activities of topical antiglaucoma agents.

Preservative-free anti-glaucoma therapy is a realistic target for the future. The evidence demonstrating that a considerable proportion of topical adverse events may result from preservatives rather than active agents is growing. Therefore, an effort to reduce the overall preservative load for patients is essential. Nevertheless, preservative-free preparations remain expensive and, for some, inconvenient to use. In the future, it is anticipated that the development of new technologies and the introduction of novel multidose preservative-free systems, such as what was used for the latanoprost/timolol eye drop evaluated in this study, will allow more patients to administer preservative-free antiglaucoma medications.

\section{CONCLUSIONS}

Overall, the present study confirmed the non-inferiority of the developed PF-latanoprost/timolol eye formulations to the BAK-preserved control formulation in lowering IOP and based on its safety profile. The new PFlatanoprost/timolol combination may offer an adequate IOP-lowering effect that is comparable to that obtained with existing preserved formulations. It may prove beneficial in cases where long-term topical treatment is required and in patients with ocular comorbidities.

\section{ETHICS DECLARATIONS}

Ethical approval: The study protocol was prospectively approved by the National (Hellenic) Ethics Committee (NEC) (October 31, 2018) and the National (Hellenic) Organization of Medicines (EOF) (October 31, 2018) and performed in compliance with the Declaration of Helsinki (2004) and Good Clinical Practice (GCP) guidelines. Before the initiation of the study, written informed consent was obtained from all participants. The study was registered in the EU Clinical Trials Register database with trial identification number 2017-004524-29.

Conflicting of interests: The study sponsor participated in the study design and interpretation of the data, writing the report, and in the decision to submit the paper for publication. Theodosiadis P., Konstas A.G., Halkiadakis I., Dimera V., Koufakis D., Georgakopoulos K., Kanonidou E., 
and Lygeros $\mathrm{M}$. were investigators in the study and participated in the conduct of the study and the review and approval of the manuscript.

\section{FUNDING}

The study and medical writing support were sponsored by Pharmathen S.A., Greece. Kalantzi, Margaritis, and Soulele are employees of Pharmathen S.A. Theodosiadis P., Konstas A.G., Halkiadakis I., Dimera V., Koufakis D., Georgakopoulos CD., Kanonidou E., and Lygeros M. were investigators in this study and received financial compensation.

\section{ACKNOWLEDGEMENTS}

The authors wish to thank the investigators for allowing their patients to be included in this clinical study.

\section{REFERENCES}

1. Polo V. Treatment of Glaucoma with the Fixed Combination of Latanoprost $0.005 \%$ and Timolol $0.5 \%$. European Ophthalmic Review. 2009;03(02):33. doi: 10.17925/eor.2009.03.02.33

2. Vicente A, Prud'homme S, Ferreira J, Abegao Pinto L, Stalmans I. Open-Angle Glaucoma: Drug Development Pipeline during the Last 20 Years (1995-2015). Ophthalmic Res. 2017;57(4):201-7. doi: 10.1159/000453527 pmid: 28076854

3. Weinreb RN, Aung T, Medeiros FA. The pathophysiology and treatment of glaucoma: a review. JAMA. 2014;311(18):1901-11. doi: 10.1001/jama.2014.3192 pmid: 24825645

4. Actis AG, Rolle T. Ocular surface alterations and topical antiglaucomatous therapy: a review. Open Ophthalmol J. 2014;8:6772. doi: 10.2174/1874364101408010067 pmid: 25317218

5. Broadway D, Hopes M. Preservative-free Treatment in Glaucoma Is a Sensible and Realistic Aim for the Future. European Ophthalmic Review. 2010;04(01):23. doi: 10.17925/eor.2010.04.01.23

6. Rosin LM, Bell NP. Preservative toxicity in glaucoma medication: clinical evaluation of benzalkonium chloride-free $0.5 \%$ timolol eye drops. Clin Ophthalmol. 2013;7:2131-5. doi: 10.2147/OPTH.S41358 pmid: 24204115

7. Schwenn O, Heckmann B, Guzy C, Miller PJ. Long-term effect of latanoprost/timolol fixed combination in patients with glaucoma or ocular hypertension: a prospective, observational, noninterventional study. BMC Ophthalmol. 2010;10:21. doi: 10.1186/1471-2415-10-21 pmid: 20825668

8. Higginbotham EJ, Feldman R, Stiles M, Dubiner H, Fixed Combination Investigative $\mathrm{G}$. Latanoprost and timolol combination therapy vs monotherapy: one-year randomized trial. Arch Ophthalmol. 2002;120(7):915-22. doi: 10.1001/archopht.120.7.915 pmid: 12096962

9. Higginbotham EJ, Olander KW, Kim EE, Grunden JW, Kwok KK, Tressler CS, et al. Fixed combination of latanoprost and timolol vs individual components for primary open-angle glaucoma or ocular hypertension: a randomized, double-masked study. Arch Ophthalmol. 2010;128(2):165-72. doi: 10.1001/archophthalmol.2009.384 pmid: 20142538

10. Stevens AM, Kestelyn PA, De Bacquer D, Kestelyn PG. Benzalkonium chloride induces anterior chamber inflammation in previously untreated patients with ocular hypertension as measured by flare meter: a randomized clinical trial. Acta Ophthalmol. 2012;90(3):e221-4. doi: 10.1111/j.1755-3768.2011.02338.x pmid 22489894

11. Lopes N, Gracitelli C, Chalita M, De Faria N. Ocular Surface Evaluation After the Substitution of Benzalkonium Chloride Preserved Prostaglandin Eye Drops by a Preservative-free Prostaglandin Analogue. Med Hypothesis Discov Innov Ophthalmol. 2019;8(1):52 6. pmid: 30923724

12. Stalmans I, Sunaric Megevand G, Cordeiro MF, Hommer A, Rossetti $\mathrm{L}$, Goni $\mathrm{F}$, et al. Preservative-free treatment in glaucoma: who, when, and why. Eur J Ophthalmol. 2013;23(4):518-25. doi: 10.5301/ejo.5000270 pmid: 23483513

13. Bagnis A, Papadia M, Scotto R, Traverso CE. Antiglaucoma drugs: The role of preservative-free formulations. Saudi J Ophthalmol. 2011;25(4):389-94. doi: 10.1016/j.sjopt.2011.08.004 pmid: 23960953

14. Medicines. Fixapost Preservative Free Eye Drops: Thea Pharmaceuticals Ltd; 2018. Available from: https://www.medicines.org.uk/emc/product/9830/smpc\#gref.

15. Baudouin C, Labbe A, Liang $H$, Pauly A, Brignole-Baudouin F. Preservatives in eyedrops: the good, the bad and the ugly. Prog Retin Eye Res. 2010;29(4):312-34. doi: 10.1016/j.preteyeres.2010.03.001 pmid: 20302969

16. Musch DC, Gillespie BW. The state of being noninferior Ophthalmology. 2006;113(1):1-2. 10.1016/j.ophtha.2005.09.015 pmid: 16389103

17. Gross RL, Sullivan EK, Wells DT, Mallick S, Landry TA, Bergamini MV. Pooled results of two randomized clinical trials comparing the efficacy and safety of travoprost $0.004 \% /$ timolol $0.5 \%$ in fixed combination versus concomitant travoprost $0.004 \%$ and timolol 0.5\%. Clin Ophthalmol. 2007;1(3):317-22. pmid: 19668487

18. Nakagawa S, Usui T, Yokoo S, Omichi S, Kimakura M, Mori Y, et al. Toxicity evaluation of antiglaucoma drugs using stratified human cultivated corneal epithelial sheets. Invest Ophthalmol Vis Sci. 2012;53(9):5154-60. doi: 10.1167/iovs.12-9685 pmid: 22695966

19. Economou MA, Laukeland HK, Grabska-Liberek I, Rouland JF. Better tolerance of preservative-free latanoprost compared to preserved glaucoma eye drops: the 12-month real-life FREE study. Clin Ophthalmol. 2018;12:2399-407. doi: 10.2147/OPTH.S176605 pmid: 30538423

20. Gehlot A, Raichandni S, Trehan N. A New Futuristic Glaucoma Therapeutic Management Paradigm. International Journal of Basic \& Clinical Pharmacology. 2015;4(2):195-7. doi: 10.5455/23192003.ijbcp20150417

21. Aptel F, Choudhry R, Stalmans I. Preservative-free versus preserved latanoprost eye drops in patients with open-angle glaucoma or ocular hypertension. Curr Med Res Opin. 2016;32(8):1457-63. doi: 10.1080/03007995.2016.1202818 pmid: 27310103

22. Baudouin C, de Lunardo C. Short-term comparative study of topical $2 \%$ carteolol with and without benzalkonium chloride in healthy volunteers. $\mathrm{Br} J$ Ophthalmol. 1998;82(1):39-42. doi: 10.1136/bjo.82.1.39 pmid: 9536878

23. Januleviciene I, Derkac I, Grybauskiene L, Paulauskaite R, Gromnickaite R, Kuzmiene L. Effects of preservative-free tafluprost on tear film osmolarity, tolerability, and intraocular pressure in previously treated patients with open-angle glaucoma. Clin Ophthalmol. 2012;6:103-9. doi: 10.2147/OPTH.S28104 pmid: 22275814

24. Hamacher T, Airaksinen J, Saarela V, Liinamaa MJ, Richter U, Ropo A. Efficacy and safety levels of preserved and preservative-free tafluprost are equivalent in patients with glaucoma or ocular hypertension: results from a pharmacodynamics analysis. Acta Ophthalmol Suppl (Oxf ). 2008;242:14-9. doi: 10.1111/j.17553768.2008.01381.x pmid: 18752510 